\title{
Establishing Equivalence of Exercise Intensities
}

\author{
Aaron Lewicke ${ }^{1,2}$ and Kenneth C. Beck ${ }^{*}, 2$ \\ ${ }^{1}$ Clarkson University, Potsdam, NY 13699, USA \\ ${ }^{2}$ Boston Scientific Corp, CRM Division, Saint Paul, MN 55112, USA
}

\begin{abstract}
Introduction: Research trials that involve testing of physiological response to exercise and that include tests from more than one institution, or tests across long time periods, may be required to include tests from different ergometer types, mainly treadmill or leg cycle ergometer. The purpose of this study was to establish equivalence of metabolic demand when comparing treadmill to cycle ergometer protocols.
\end{abstract}

Methods: Published equations were used to derive external power output performed on a cycle ergometer and treadmill to match external power output performance across the range of typical body weights for subjects.

Results: When comparing a submaximal walking versus 10-watt incremental cycling protocol, the percent difference in metabolic demand ranged from $-35 \%$ to $39 \%$ across the range of body weights from $50 \mathrm{~kg}$ to $150 \mathrm{~kg}$. For the modified Bruce treadmill protocol, watts on the cycle had to be increased to match metabolic demand as body weight of the subject increases.

Conclusion: To match estimated external power output performed on a treadmill, external power output on a leg cycle ergometer must be increased as body weight increases.

Keywords: Cycle ergometry, treadmill, metabolic demand, clinical exercise testing, peak $\mathrm{VO}_{2}$.

\section{INTRODUCTION}

For research trials involving testing of the physiological response to exercise, there may be cases when different exercise equipment must be used for different individuals or testing centers. When running a clinical study a broad spectrum of individuals is desired, but a uniform testing protocol may not be possible because some individuals cannot walk on a treadmill and a stationary exercise cycle must be used instead. Other times a clinical study may involve multiple centers and some centers may only test with a stationary cycle, while other centers may test using a treadmill. A few studies have compared testing with a cycle to a treadmill for prognostic markers of coronary artery disease [1-3]. In these cases, matching the external power output is important, as the external power output imposed dictates the stress imposed on the cardio-pulmonary and musculo-skeletal systems during the testing [4]. Can exercise protocols be designed to be equivalent across testing modalities, and thus minimizing differences in cardiovascular risk in patients?

Exercise protocols are usually defined as settings of an ergometer (treadmill or cycle) at set times during the protocol. For instance, the Bruce protocol uses a treadmill and 3 minute stages, where after each 3 minute period both speed and elevation are increased [5]. In clinical testing, cycle protocols are often tailored to the patient, and usually involve 1 to 3 minute stages with incremental changes in power output of the cycle, typically in 10 to 40 watt increments. The metabolic demand and therefore cardiopulmonary stress

*Address correspondence to this author at the Boston Scientific CRM, 4100 Hamline Ave N, St Paul, MN 55112, USA; Tel: 651582 6602; Fax: 651 582 2872; E-mail: kenneth.beck@bsci.com imposed on the subject by such protocols will depend on the external power output. For large research trials, it may be useful to design protocols with similar stress profiles on both treadmill and exercise cycle.

In this study, published equations were used to compare submaximal exercise responses on a treadmill to a cycle ergometer and to establish equivalent external power outputs over various body weights.

\section{METHODS}

To estimate the metabolic demand associated with exercise, one must calculate the amount of external work performed by skeletal muscles, and then convert that to metabolic cost. Quantifying external work performed is relatively easy for cycle ergometry, but more difficult for treadmills due to unpredictable factors such as gait efficiency and varying center of gravity as the angle of the treadmill is changed [4]. To quantify energy consumed by working muscles, oxygen consumption is measured and caloric expenditure is derived, since the calorie is the basic unit of energy or work. This conversion depends on the type of metabolic fuel used, but a generally held conversion that averages across carbohydrates, fats, and proteins as fuels is $4.83 \mathrm{Cal} /$ liter of $\mathrm{O}_{2}$ [4]. However, this equation only tells us the metabolic energy expenditure since the external power output performed by the muscles is far less as the muscles are at most about $30 \%$ efficient [6]. To convert from external power output performed to metabolic demand, data derived from populations of subjects studied with standard protocols was used.

For cycle ergometer testing, the external power output can be known exactly and is roughly independent of body weight, since calculating work is a simple task of measuring 
torque on the pedals and pedal speed. To determine the metabolic cost of external power output during an exercise test using a stationary exercise cycle, the following empirical equation is used [5]:

$$
V_{O 2} / B W=1.8 * 6.12 *(W A T T S) / B W+3.5+3.5
$$

where BW is body weight in kilograms, WATTS is the ergometer power output, 6.12 is used to convert from watts to $\mathrm{kg} \cdot \mathrm{m} \cdot \mathrm{min}^{-1}$, and the units of $\dot{V}_{O 2} / B W$ are in $\mathrm{mL} \cdot \mathrm{kg}^{-1} \cdot \mathrm{min}^{-1}$. In this equation, the first term is the metabolic cost of performing the external power output turning the pedals against resistnace, in watts, the first " 3.5 " term represents resting metabolic energy expenditure, and the second " 3.5 " term accounts for the metabolic cost of turning the legs against no force on the pedals. A refinement of this equation would include the increase in metabolic cost of turning the legs with increased leg mass [7] and addition of several other internal sites of oxygen consumption such as postural and respiratory muscle activity. However, for simplicity leg mass and internal oxygen consumption have not been included in the cycle ergometer calculations of this study.

The calculations for treadmills are more involved. There are multiple components of external power output performed when walking or running on a treadmill: energy cost of level walking, energy cost of level running, and energy cost of work against gravity on a sloping treadmill. The first two components are highly dependent on walking or running style and efficiency, and cannot be easily predicted precisely on first principles. The last component, work against gravity, increases with inclination of the treadmill. Because of the uncertainty of the first three components, generalized empirical equations are used.

For a walking on a treadmill with an incline the $V_{O 2} / B W$ consumed is given by [5]:

$V_{O 2} / B W=0.1 * 26.82 *(M P H)+1.8 * G * 26.82 *(M P H)+3.5$

Similarly, for running on a treadmill with an incline the $V_{O 2} / B W$ consumed is given by [5]:

$V_{O 2} / B W=0.2 * 26.82 *(M P H)+0.9 * G * 26.82 *(M P H)+3.5$

where MPH is the speed of the treadmill in miles per hour, $\mathrm{G}$ is the grade of the treadmill, 26.82 is to convert from mph to meters per minute, and the units of $V_{O 2} / B W$ are in $\mathrm{mL} \cdot \mathrm{kg}^{-1} \cdot \mathrm{min}^{-1}$.

The horizontal component of work, which is the first term in equations 2 and 3 , is dependent on body weight, speed, and walking $v s$ running. Note that the metabolic cost of running on the level $(\mathrm{G}=0)$, is about twice that of walking for the same speed, though generally the speeds do not overlap. Also note that the work done against gravity, the second term in equations 2 and 3, is twice as much for walking as it is for running. The final constant is the metabolic demand while at rest.

The stated equations were derived from steady-state aerobic exercise and could exhibit inter-subject variability upwards of 7\% [5]. Thus, judicious use of these equations is required as exercise responses could be affected by age, gender, and training status.

A walking exercise protocol was compared to a leg cycle ergometer protocol, starting with a low stress, submaximal protocol. The cycle "equivalent" is a 10-watt incremental protocol. The two protocols are compared for body weights ranging from that for a petite person $(50 \mathrm{~kg})$ up to moderately obese $(150 \mathrm{~kg})$. For comparison, the \% difference in METS was calculated using the equation

$$
\text { METS \% Difference }=100 * \frac{(\text { Cycle }- \text { Walking })}{\left(\frac{\text { Cycle }+ \text { Walking }}{2}\right)}
$$

\section{RESULTS}

Table 1 illustrates a treadmill protocol that involves level walking in $1 \mathrm{MPH}$ increments up to the point where most people would break into a jog, 3.5 to $4 \mathrm{MPH}$. The lowest intensity on the cycle is always higher metabolic stress compared to $1 \mathrm{MPH}$ on the treadmill. The petite person would be working relatively harder on the cycle throughout this protocol, whereas the heavier person would have higher metabolic stress at the faster walking speeds on the treadmill.

In Table 2, the metabolic stress during a standard treadmill protocol, the modified Bruce $[8,9]$, was matched to the cycle ergometer. To simplify calculations, only the equation for walking on a treadmill with stopping at a speed of 4.2 $\mathrm{mph}$ was considered. By re-arranging equations 2 and 3 the watts on the cycle are adjusted in each stage to match the METS on the treadmill. For the first stage of the Bruce, the power setting on the cycle would be less than what most stationary leg cycle ergometers are capable of (internal resistance is often about 8-15 watts). The second through last stages can be matched, but the power output required on a cycle increases with body weight to match metabolic demand on the treadmill.

\section{DISCUSSION}

In this study, it has been shown that when attempting to match exercise intensity between cycle ergometry and treadmill exercise, if intensity is not adjusted on a cycle ergometer to compensate for body weight there are large differences in metabolic requirements between a treadmill and cycle ergometer. Rahimi et al. evaluated many prognostic markers for coronary artery disease for both the cycle and treadmill concluding that risk factors for cycle exercise tests need to be reevaluated. They found differences in heart rate responses, systolic blood pressure, chronotropic incompetence, and Duke treadmill score [1]. Klein et al. compared treadmill and cycle exercise responses in 140 patients and concluded that cycle and treadmill evoke different hemodynamic and clinical responses [2]. Hambrecht et al. studied the sensitivity of cycle and treadmill for detecting coronary artery disease and concluded that the treadmill has a greater ability for detection [3].

When addressing the risk assessment for a clinical study that involves multiple centers with multiple testing modalities, the metabolic and cardiopulmonary stress increases with body weight on treadmills, but not as much on a leg cycle ergometer. Also, when considering the ramifications of test- 
Table 1. A Treadmill Protocol Compared to a Leg Cycle Ergometer Protocol

\begin{tabular}{|c|c|c|c|c|c|c|c|}
\hline \multirow[b]{2}{*}{ BW } & \multicolumn{2}{|c|}{ Walking $0 \%$ Grade } & \multirow[b]{2}{*}{ METS } & \multicolumn{2}{|c|}{ Cycle Ergometer } & \multirow[b]{2}{*}{ METS } & \multirow[b]{2}{*}{ Cycle-Tread \% Diff } \\
\hline & mph & $\mathrm{VO}_{2} / \mathrm{kg}$ & & Watts & $\mathrm{VO}_{2} / \mathrm{kg}$ & & \\
\hline 50 & 1 & 6.18 & 1.77 & 10 & 9.21 & 2.63 & 39.4 \\
\hline 50 & 2 & 8.86 & 2.53 & 20 & 11.42 & 3.26 & 25.2 \\
\hline 50 & 3 & 11.55 & 3.30 & 30 & 13.64 & 3.90 & 16.6 \\
\hline 50 & 4 & 14.23 & 4.07 & 40 & 15.85 & 4.53 & 10.8 \\
\hline 75 & 1 & 6.18 & 1.77 & 10 & 8.47 & 2.42 & 31.3 \\
\hline 75 & 2 & 8.86 & 2.53 & 20 & 9.95 & 2.84 & 11.5 \\
\hline 75 & 3 & 11.55 & 3.30 & 30 & 11.42 & 3.26 & -1.1 \\
\hline 75 & 4 & 14.23 & 4.07 & 40 & 12.90 & 3.69 & -9.8 \\
\hline 100 & 1 & 6.18 & 1.77 & 10 & 8.11 & 2.32 & 26.9 \\
\hline 100 & 2 & 8.86 & 2.53 & 20 & 9.21 & 2.63 & 3.9 \\
\hline 100 & 3 & 11.55 & 3.30 & 30 & 10.32 & 2.95 & -11.2 \\
\hline 100 & 4 & 14.23 & 4.07 & 40 & 11.42 & 3.26 & -21.9 \\
\hline 125 & 1 & 6.18 & 1.77 & 10 & 7.88 & 2.25 & 24.2 \\
\hline 125 & 2 & 8.86 & 2.53 & 20 & 8.77 & 2.51 & -1.1 \\
\hline 125 & 3 & 11.55 & 3.30 & 30 & 9.65 & 2.76 & -17.8 \\
\hline 125 & 4 & 14.23 & 4.07 & 40 & 10.54 & 3.01 & -29.8 \\
\hline 150 & 1 & 6.18 & 1.77 & 10 & 7.74 & 2.21 & 22.4 \\
\hline 150 & 2 & 8.86 & 2.53 & 20 & 8.47 & 2.42 & -4.5 \\
\hline 150 & 3 & 11.55 & 3.30 & 30 & 9.21 & 2.63 & -22.5 \\
\hline 150 & 4 & 14.23 & 4.07 & 40 & 9.95 & 2.84 & -35.4 \\
\hline
\end{tabular}

Where $\mathrm{BW}$ is body weight, $\mathrm{mph}$ is miles per hour, $\mathrm{VO}_{2} / \mathrm{kg}$ is the volume of oxygen consumed per minute per kilogram, and METS is metabolic equivalents.

ing modalities it is important to consider the study design. If a study assesses changes in $\dot{V}_{\mathrm{O} 2} / \mathrm{kg}$ at submaximal efforts in repeat visits, it is very important to assure that the workloads the individuals experience are similar throughout the course of the study. If a multi-center study involves crosssectional differences between multiple study populations, then it is also important to consider the exercise test modality employed. Adjusting workloads in both of these situations will assure the quality of the exercise test data for cross sectional comparisons.

Controlling exercise workloads is necessary to achieve an optimal rate of work increase to reach $\mathrm{Max} V_{\mathrm{O}_{2}}$ or peak $\dot{V}_{O 2}$ in approximately $10 \mathrm{~min}$ [4]. Matching the incremental increase of energy expenditure per stage is also important for comparing across multiple visits, or different exercise modalities. Thus, even in cases where metabolic responses are being measured directly, protocols should still be matched to optimize duration of exercise.

For example, assume a large clinical study is being designed that will include centers in the U.S. (where treadmill testing is used) and Europe (using cycle ergometry). For the U.S. centers, a standard protocol is usually chosen, such as the Naughton, Bruce or modified Bruce [5]. To minimize differences in metabolic and cardiac stress between the two geographies, it would be recommended to use cycle protocols that depend on body weight, such as presented in Table 2.

The equations used in this work were derived from those published in the ACSM Manual for exercise testing and prescription [5]. These equations were derived from studies of large numbers of normal individuals. Thus, the parameters in the equations are averages across the population, and there is variability (as high as 7\%) in these values across individuals in the population [5].

In addition, the equations rely heavily on the normalization of $\dot{V}_{O 2}$ for body weight, a normalization that is imperfect but widely used. Thus, the equivalence tables presented are approximations at best, but adjustment of protocols using these values would be better than using fixed power output increments in patients of differing body weights. Further research is needed to derive the equations in patient populations, and to add work of breathing, differences in cycle efficiency over the range of body weights and other secondary factors.

\section{CONCLUSION}

There are a few simple mitigations for energy consumption differences in studies requiring testing with a mix of treadmill and leg cycle ergometry: 1) require only treadmill 
Table 2. Cycle Watts Adjusted to Match METS from a Modified Bruce Protocol

\begin{tabular}{|c|c|c|c|c|c|c|c|}
\hline \multirow{2}{*}{ BW } & \multicolumn{4}{|c|}{ Modified Bruce Treadmill } & \multicolumn{3}{|c|}{ Cycle Ergometer } \\
\hline & mph & Elevation & $\mathrm{VO}_{2} / \mathbf{k g}$ & METS & Watts & $\mathrm{VO}_{2} / \mathbf{k g}$ & METS \\
\hline 50 & 1.7 & $0 \%$ & 8.06 & 2.30 & 5 & 8.11 & 2.30 \\
\hline 50 & 1.7 & $5 \%$ & 12.16 & 3.48 & 24 & 12.39 & 3.48 \\
\hline 50 & 1.7 & $10 \%$ & 16.27 & 4.65 & 44 & 16.67 & 4.65 \\
\hline 50 & 2.5 & $12 \%$ & 24.69 & 7.05 & 83 & 25.46 & 7.05 \\
\hline 50 & 3.4 & $14 \%$ & 35.60 & 10.17 & 135 & 36.84 & 10.17 \\
\hline 50 & 4.2 & $16 \%$ & 47.21 & 13.49 & 190 & 48.95 & 13.49 \\
\hline 75 & 1.7 & $0 \%$ & 8.06 & 2.30 & 7 & 8.11 & 2.30 \\
\hline 75 & 1.7 & $5 \%$ & 12.16 & 3.48 & 37 & 12.39 & 3.48 \\
\hline 75 & 1.7 & $10 \%$ & 16.27 & 4.65 & 66 & 16.67 & 4.65 \\
\hline 75 & 2.5 & $12 \%$ & 24.69 & 7.05 & 125 & 25.46 & 7.05 \\
\hline 75 & 3.4 & $14 \%$ & 35.60 & 10.17 & 202 & 36.84 & 10.17 \\
\hline 75 & 4.2 & $16 \%$ & 47.21 & 13.49 & 284 & 48.95 & 13.49 \\
\hline 100 & 1.7 & $0 \%$ & 8.06 & 2.30 & 10 & 8.11 & 2.30 \\
\hline 100 & 1.7 & $5 \%$ & 12.16 & 3.48 & 49 & 12.39 & 3.48 \\
\hline 100 & 1.7 & $10 \%$ & 16.27 & 4.65 & 87 & 16.67 & 4.65 \\
\hline 100 & 2.5 & $12 \%$ & 24.69 & 7.05 & 167 & 25.46 & 7.05 \\
\hline 100 & 3.4 & $14 \%$ & 35.60 & 10.17 & 270 & 36.84 & 10.17 \\
\hline 100 & 4.2 & $16 \%$ & 47.21 & 13.49 & 379 & 48.95 & 13.49 \\
\hline 125 & 1.7 & $0 \%$ & 8.06 & 2.30 & 12 & 8.11 & 2.30 \\
\hline 125 & 1.7 & $5 \%$ & 12.16 & 3.48 & 61 & 12.39 & 3.48 \\
\hline 125 & 1.7 & $10 \%$ & 16.27 & 4.65 & 109 & 16.67 & 4.65 \\
\hline 125 & 2.5 & $12 \%$ & 24.69 & 7.05 & 209 & 25.46 & 7.05 \\
\hline 125 & 3.4 & $14 \%$ & 35.60 & 10.17 & 337 & 36.84 & 10.17 \\
\hline 125 & 4.2 & $16 \%$ & 47.21 & 13.49 & 474 & 48.95 & 13.49 \\
\hline 150 & 1.7 & $0 \%$ & 8.06 & 2.30 & 15 & 8.06 & 2.30 \\
\hline 150 & 1.7 & $5 \%$ & 12.16 & 3.48 & 73 & 12.16 & 3.48 \\
\hline 150 & 1.7 & $10 \%$ & 16.27 & 4.65 & 131 & 16.27 & 4.65 \\
\hline 150 & 2.5 & $12 \%$ & 24.69 & 7.05 & 250 & 24.69 & 7.05 \\
\hline 150 & 3.4 & $14 \%$ & 35.60 & 10.17 & 405 & 35.60 & 10.17 \\
\hline 150 & 4.2 & $16 \%$ & 47.21 & 13.49 & 569 & 47.21 & 13.49 \\
\hline
\end{tabular}

Where $\mathrm{BW}$ is body weight, $\mathrm{mph}$ is miles per hour, $\mathrm{VO}_{2} / \mathrm{kg}$ is the volume of oxygen consumed per minute per kilogram, and METS is metabolic equivalents.

or only leg cycle ergometry; 2) test a patient population of narrow range of body weights; 3) allow the exercise test workload on the leg cycle ergometer to be dependent on a patient's body weight, as demonstrated in Table 2.

\section{ACKNOWLEDGEMENTS}

The results of the present study do not constitute endorsement by ACSM.

This work was performed at Boston Scientific CRM, St Paul, MN, USA.

\section{REFERENCES}

[1] Rahimi K, Thomas A, Adam M, Hayerizadeh BF, Schuler G, Secknus MA. Implications of exercise test modality on modern prognostic markers in patients with known or suspected coronary artery disease: treadmill versus bicycle. Eur J Cardiovasc Prev Rehabil 2006; 13(1): 45-50.

[2] Klein J, Cheo S, Berman DS, Rozanski A. Pathophysiologic factors governing the variability of ischemic responses to treadmill and bicycle exercise. Am Heart J 1994; 128(5): 948-55.

[3] Hambrecht RP, Schuler GC, Muth T, et al. Greater diagnostic sensitivity of treadmill versus cycle exercise testing of asymptomatic men with coronary artery disease. Am J Cardiol 1992; 70(2): 141-6. 
[4] Wasserman K. Principles of exercise testing and interpretation : including pathophysiology and clinical applications. Philadelphia: Lippincott Williams \& Wilkins 2005; pp. 3-19.

[5] American College of Sports Medicine. Whaley MH, Brubaker PH, Otto RM, Armstrong LE, Eds. ACSM's guidelines for exercise testing and prescription. 7th ed. Philadelphia, PA: Lippincott Williams \& Wilkins 2006; pp. 286-89.

[6] Whipp BJ, Wasserman K. Efficiency of muscular work. J Appl Physiol 1969; 26(5): 644-8.
[7] Wasserman K, Whipp BJ. Excercise physiology in health and disease. Am Rev Respir Dis 1975; 112(2): 219-49.

[8] Handler CE, Sowton E. A comparison of the Naughton and modified Bruce treadmill exercise protocols in their ability to detect ischaemic abnormalities six weeks after myocardial infarction. Eur Heart J 1984; 5(9): 752-5.

[9] McInnis KJ, Balady GJ, Weiner DA, Ryan TJ. Comparison of ischemic and physiologic responses during exercise tests in men using the standard and modified Bruce protocols. Am J Cardiol 1992; 69(1): 84-9.

(C) Lewicke and Beck; Licensee Bentham Open

This is an open access article licensed under the terms of the Creative Commons Attribution Non-Commercial License (http://creativecommons.org/licenses/by$\mathrm{nc} / 3.0 /$ ) which permits unrestricted, non-commercial use, distribution and reproduction in any medium, provided the work is properly cited. 